\title{
Synthesis and characterization of polyurethane microspheres
}

\author{
L.S. Ramanathan, P. G. Shukla And S. Sivaram \\ Division of Polymer Chemistry, National Chemical Laboratory, Pune 411008, India

\begin{abstract}
A novel particle forming polymerization technique for the preparation of polyurethane microspheres with particle size in the range $0.1-100$ micron is described. The method is general, applicable to wide variety of diols and isocyanates and is very simple. The key to successful particle forming polymerization is the use of novel steric stabilizers, such as, a reactive diol containing two primary hydroxyl groups with a long hydrophobic acrylate ester moiety and an amphiphilic block copolymer. The effect of various reaction variables on the particle forming polymerization process will be discussed.
\end{abstract}

\section{INTRODUCTION}

Preparation of polymers in particulate form has been well studied using techniques such as suspension, emulsion and dispersion polymerization. Of these, dispersion polymerization has been found to be an efficient method for producing monodisperse polymer particles. A comparison of various techniques employed for producing nano and microspheres have been reviewed (ref.1) Particle forming polymerization process reported so far are typically performed with vinyl monomers (ref 2-4). There is, however, no report on the particle forming polymerization of an isocyanate with a diol to produce polyurethane microspheres. Conventionally, polyurethane particles can be prepared by cryogenic grinding of thermoplastic polyurethanes (ref.5) or by suspension polymerization of isocyanate terminated prepolymers in aqueous or non-aqueous medium (ref.6). However, in none of these prior methods the control of particle size has been demonstrated. In this paper, we report for the first time, the synthesis of polyurethane microspheres by particle forming polymerization techniques using a novel polycondensable macromonomer (1) (ref. 7) and an amphiphilic block copolymer (2) as steric stabilizers.<smiles>CCC(CO)(CO)COC(=O)CSCC(C)(C)C</smiles>

(1)

1a: $\mathrm{n}=3.0$

$1 \mathrm{~b}: \mathrm{n}=6.6$

1c: $n=18.6$<smiles>[R]C=CCCCCCOCC</smiles>

(2)

2a: $\mathrm{n}=57 ; \mathrm{m}=19$

$2 \mathbf{b}: \mathrm{n}=92 ; \mathrm{m}=50$

2c: $n=463 ; m=79$

\section{EXPERIMENTAL}

\section{$\underline{\text { Materials }}$}

2-ethyl 1,3-hexanediol, (EHG) (Fluka), ethylene glycol (EG) (s.d.fine, India), toluene diisocyanate (TDI), dibutyl tin dilaurate (DBTDL) (E merck), diazo bicyclo [2,2,2] octane (DABCO) and paraffin oil heavy, (s.d.fine, India) were used as received.

\section{Synthesis of polyurethane microparticles by dispersion polymerization:}

In a four neck $250 \mathrm{~mL}$ round bottom flask fitted with a stirrer, condenser, nitrogen inlet and a thermowell, $0.34 \mathrm{~g}$ macrodiol (1 $), 0.5 \mathrm{~g}$ of $1 \% \mathrm{DBTDL}$ in paraffin oil and $25 \mathrm{~g}$ paraffin oil were added and stirred at $500 \mathrm{rpm}$. The mixture was heated to $65^{\circ} \mathrm{C}$ and $3 \mathrm{~g}$ EHG and $4.6 \mathrm{~g}$ TDI were added. The reaction was continued for $5 \mathrm{~h}$. The polyurethane microspheres formed were washed with hexane to remove paraffin oil 
and is dried under vacuum at room temperature for $4 \mathrm{~h}$ to get free flowing polyurethane microspheres. Yield: $90 \%$; IR (KBr): $3400 \mathrm{~cm}^{-1}$ (-NH); $1750 \mathrm{~cm}^{-1}$ (-COO); $1710 \mathrm{~cm}^{-1}$ (-NHCOO-); ${ }^{1} \mathrm{H}$ NMR (actone $\left.\mathrm{D}_{6}\right): \delta=7.1-8.0\left(\mathrm{~m} ;\right.$-aromatic); 4.1-4.3(br; $\left.-\mathrm{COOCH}_{2}\right) ; 1.1-1.5\left(\mathrm{br} ; \mathrm{CH}_{2}\right) ; 0.75-0.8\left(\mathrm{t} ;-\mathrm{CH}_{2}-\mathrm{CH}_{3}\right)$

\section{Synthesis of polyurethane microparticles by suspension polymerization:}

In a four neck $250 \mathrm{~mL}$ round bottom flask fitted with a stirrer, condenser, nitrogen inlet and a thermowell, $0.15 \mathrm{~g}$ block copolymer $(\underline{\mathbf{b}}), 0.5 \mathrm{~g}$ of $\mathrm{DABCO}, 3 \mathrm{~g} \mathrm{EG}$ and $50 \mathrm{~g}$ paraffin oil were added and stirred at 1000 $\mathrm{rpm}$. The mixture was heated to $65^{\circ} \mathrm{C}$ and $10.6 \mathrm{~g}$ TDI was added. The reaction was continued for $5 \mathrm{~h}$. The polyurethane microspheres formed was washed with hexane to remove paraffin oil and is dried under vacuum at room temperature for $4 \mathrm{~h}$ to get free flowing polyurethane microspheres. Yield: $65 \%$; IR (KBr): $3400 \mathrm{~cm}^{-1}(-\mathrm{NH}) ; 1750 \mathrm{~cm}^{-1}(-\mathrm{COO}) ; 1710 \mathrm{~cm}^{-1}$ (-NHCOO-).

\section{Measurements:}

Hydroxyl values of the macrodiols were determined according to ASTM procedure D1957. [Annual book of ASTM standards vol. 06.03, sec 6 (1991)]. Molecular weight of the macrodiol was determined by KNAUER vapour pressure osmometer in $\mathrm{CHCl}_{3}$ at $35^{\circ} \mathrm{C}$. IR spectra were recorded on a Perkin Elmer 16 PC FT-IR spectrometer. ${ }^{1} \mathrm{H}$ NMR spectra were recorded on a $200 \mathrm{MHz}$ Bruker AC $200 \mathrm{NMR}$ spectrometer. Particle size and size distributions were measured using a Malvern Photon Correlation Photometer, model 4700 using a vertically polarized $25 \mathrm{mw} \mathrm{He}-\mathrm{Ne}$ laser as light source.

\section{RESULTS AND DISCUSSION}

The macrodiols were prepared by the reaction of TMP with a carboxyl terminated poly (lauryl methacrylate) (ref.7). The properties of macrodiol are shown in Table 1.

TABLE 1: Properties of Macrodiols.

\begin{tabular}{|l|l|l|l|l|}
\hline Macrodiol & $\begin{array}{c}\text { Hydroxyl } \\
\text { value } \\
\text { (mg } \\
\text { KOH/g) }\end{array}$ & \multicolumn{2}{|c|}{$\overline{\mathrm{M}}_{\mathrm{n}}$} & $\overline{\mathrm{f}}_{\mathrm{n}}$ \\
\hline & & $\begin{array}{l}\text { By end } \\
\text { group. }\end{array}$ & By VPO & \\
\hline 1a & 157.2 & 710 & 760 & 1.87 \\
\hline 1b & 75.5 & 1490 & 1670 & 1.78 \\
\hline 1c & 26.08 & 4300 & 4720 & 1.82 \\
\hline
\end{tabular}

The observed functionality indicates the near quantitative presence of the 1,3-glycol moiety in the macrodiol.

\section{Synthesis of PU microspheres using macrodiol as steric stabilizer.}

Dispersion polymerization of EHG and TDI was carried out in paraffin oil in the presence of different amounts of macrodiol stabilizers (Table 2). With macrodiol $1 \mathrm{a}$, no dispersion could be obtained as the whole mass coagulated. However, with macrodiol $1 \mathbf{b}$ and $1 \mathrm{c}$, stable dispersion could be obtained at the specified concentration of the stabilizer. The particle size histogram is given in Fig. 1 which indicate that nearly monodisperse particles in nanometer size are obtained when $10 \mathrm{wt} \%$ of macrodiol $1 \mathrm{c}$ was used as a stabilizer. As the concentration is increased from 10 to $15 \mathrm{wt} \%$ there is no significant change either in particle size or particle size distribution. When the concentration is decreased from 10 to $5 \mathrm{wt} \%$, micron size polyurethane particles are formed with broad particle size distribution. 
TABLE 2: Properties of polyurethane particles obtained using macrodiol stabilizers.

\begin{tabular}{|c|l|l|l|l|}
\hline $\begin{array}{c}\text { Macrodiol } \\
\text { stabilizer }\end{array}$ & $\begin{array}{l}\text { Concentration } \\
\text { Wt } \%\end{array}$ & $\begin{array}{c}\text { Particle } \\
\text { size } \\
(\mathrm{nm})\end{array}$ & $\mathrm{D}_{\mathrm{w}} / \mathrm{D}_{\mathrm{n}}$ & \% Yield \\
\hline 1a & 15 & \multicolumn{3}{|c|}{ Coagulated } \\
\hline 1b & 5 & \multicolumn{3}{|c|}{ Partially agglomerated } \\
\hline & 10 & - & - & 88 \\
\hline & 15 & 1400 & 1.30 & 93 \\
\hline & 5 & - & - & 93 \\
\hline c & 10 & 220 & 1.16 & 95 \\
\hline & 15 & 180 & 1.24 & 95 \\
\hline \multicolumn{4}{|l|}{ based on isocyanate forming reactants. } \\
\hline
\end{tabular}

In the case of macrodiol $\underline{\mathbf{1 b}}$, as the concentration of the stabilizer is increased, the particle size decreases. Nevertheless, the particle size distribution was broad even at higher stabilizer concentration. A control experiment without macrodiol stabilizer did not produce polyurethane in a particulate form. The IR spectrum of the polyurethane particle shows both an ester $\left(1775 \mathrm{~cm}^{-1}\right)$ and an urethane $\left(1710 \mathrm{~cm}^{-1}\right)$ group. This observation indicates that the macrodiol stabilizer participates in the urethane forming reaction. The pendant hydrophobic PLMA moiety stabilizes the polyurethane particle formed by steric stabilization mechanism. This explanation is in accordance with the proposed theories of particle forming mechanism (ref 8). The decrease in particle size of the polyurethane dispersion with an increase in macrodiol concentration can be understood based on the premise that in dispersion polymerization nucleation takes place at the early stages where the particle number reaches its final value (ref.9). So, higher stabilizer concentration prevents coagulation of the initially formed nuclei and reduces the particle size. A similar phenomenon was observed in the radical dispersion polymerization using a macrodiol stabilizer (ref.4 and $10)$.
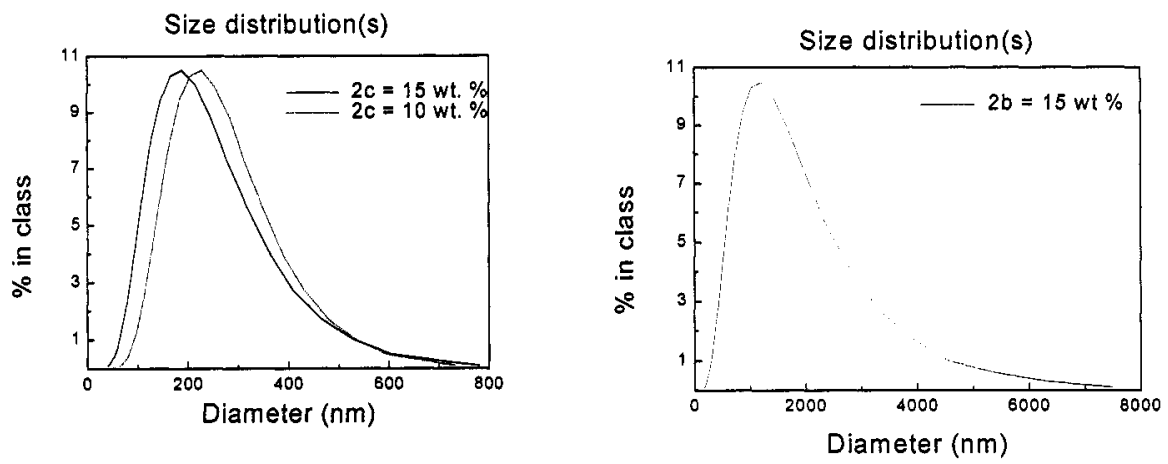

Fig 1: Effect of molecular weight and concentration of macrodiol stabilizer on particle size and size distribution of polyurethane particles.

\section{Svnthesis of PU microspheres using an amphiphilic block copolymer as steric stabilizer.}

Suspension polymerization of EG and TDI was carried out using a block copolymer, namely, poly (butadiene-b-ethylene oxide) as steric stabilizer. The block copolymer was prepared by a sequential anionic polymerization technique. The properties of polyurethane particles prepared from such a process are shown in Table 3. Poly (butadiene-b-ethylene oxide) was chosen as steric stabilizer because it contains an hydrophilic anchor block (PEO segment) and freely soluble stabilizing moiety (poly butadiene segment) in the dispersion medium. Hence it should stabilize the polyurethane particles effectively according to the 
principles of steric stabilization (ref.8). As the ratio of anchor to stabilizing moiety ratio increases, the particle size decreases. However when the ratio is 7.14 , there is an increase in particle size. This could be due to the poor solubility of $\underline{\mathbf{c}}$ in paraffin oil, because of higher PEO segment length.

TABLE 3: Characteristics of poly (butadiene-b-ethylene oxide) copolymer.

\begin{tabular}{|l|l|l|l|l|l|}
\hline Sample & \multicolumn{2}{|l|}{$\mathrm{M}_{\mathfrak{n}}$} & $\begin{array}{l}\text { Anchor/ } \\
\text { Stabilizing } \\
\text { moiety } \\
\text { ratio }\end{array}$ & \multicolumn{2}{l|}{$\begin{array}{l}\text { Properties of PU } \\
\text { particles }\end{array}$} \\
\hline & & & $\begin{array}{l}\text { particle } \\
\text { size }\end{array}$ & $\mathrm{D}_{\mathrm{w}} / \mathrm{D}_{\mathrm{n}}$ \\
\hline & $\begin{array}{l}\text { Poly } \\
\text { butadiene } \\
\text { segment }\end{array}$ & $\begin{array}{l}\text { PEO } \\
\text { segment }\end{array}$ & & & \\
\hline $\mathbf{2 a}$ & 3100 & 850 & 3.64 & 13 & 1.68 \\
\hline $\mathbf{2 b}$ & 5000 & 2200 & 2.27 & 22 & 1.7 \\
\hline $\mathbf{2 c}$ & 25000 & 3500 & 7.14 & 50 & - \\
\hline
\end{tabular}

The effect of stabilizer concentration on particle size and size distribution was carried out using block copolymer 2b (Fig 2). An increase in stabilizer concentration has no significant effect on particle size. Nevertheless, as the stabilizer concentration increases, a change from bimodal to unimodal size distribution was observed. Here, the PU particle formed is stabilized mainly by the adsorption of the amphiphilic block copolymer on the particle surface. So, at higher stabilizer concentration, the amount of stabilizer adsorbed on the particle surface is more and hence stabilizes the particle effectively.

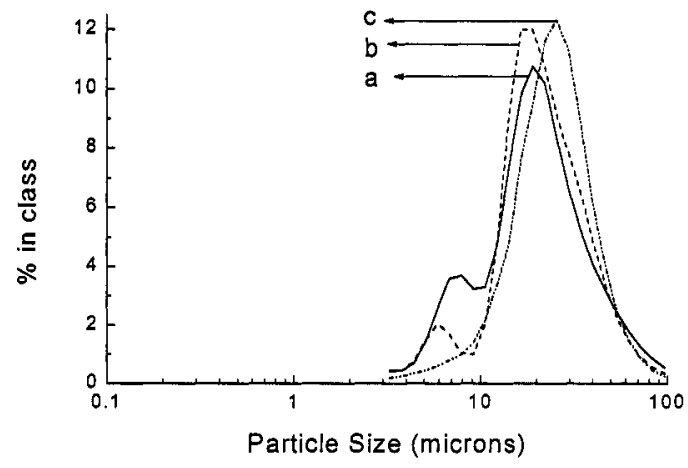

Fig.2 Effect of stabilizer concentration on particle size and particle size distribution of PU microspheres prepared using poly (butadiene-b-ethylene oxide) as steric stabilizer.

(a) Stabilizer Concentration $=0.75 \%$; Particle Size $=21.8 \mu ; D_{w} / D_{n}=2.0$

(b) Stabilizer Concentration $=1.50 \%$; Particle Size $=21.8 \mu ; D_{\mathrm{w}} / \mathrm{D}_{\mathrm{n}}=1.7$

(c) Stabilizer Concentration $=4.50 \% ;$ Particle Size $=24 \mu ; D_{w} / D_{n}=1.36$

\section{CONCLUSION}

A novel polycondensable macrodiol with a long hydrophobic acrylate ester moiety and an amphiphilic block copolymer were successfully used in the particle forming polymerization of diisocyanate and diol. The performance of the macrodiol and the block copolymer as stabilizers depends both on its molecular weight and concentration. The particle size decreases as the concentration of the stabilizer increases and nearly monodisperse polyurethane microspheres are formed in nanometer size range in the case of macrodiol stabilizer. This new class of macrodiol steric stabilizer participates in the urethane forming reaction and is enchained in the polymer. Further studies are in progress to fully elucidate the mechanism 
of stabilization and compare the efficiency of steric stabilization by polymerizable stabilizer with a conventional steric stabilizer which functions solely by an adsorption mechanism.

\section{ACKNOWLEDGEMENT}

The authors would like to thank Prof. G. Riess, E N S de chemie, Mulhouse, France for the block copolymer samples. LSR would like to express his gratitude to Council of Scientific Industrial Research (CSIR), New Delhi, India, for the award of a Senior Research fellowship.

\section{REFERENCES}

1. R. Arshady, J. Microencapsulation, 5, 101 (1980)

2. S.Shen, E.D. Sudol and M.S. El-Aasser, J. Polym. Sci, Polym. Chem. Ed. 31, 1393 (1993)

3. S. Kobayashi, H.Uyama, J.H.Choi and Y.Matsumoto, Polym. Int., 30, 265, (1993)

4. E. Bourgeat-Lami and A. Guyot, Colloid Polym. Sci., 275, 716, (1997)

5. U.S.Patent 3214411, J. H. Sounders, (1965)

6. U.S.Patent, 4,083,831, Amrstorng Cork. Co., (1989); U.S.Patent 4,032,516, USM Corp., (1975).

7. L. S. Ramanathan and S. Sivaram Communicated to Macromolecules.

8. K.E.J. Barrett, Dispersion polymerization in organic media, Wiley, London (1975)

9. S. Kawaguchi, M.A.Winnik, and K.Ito Macromolecules, 28, 1159, (1995)

10. S. Kobayashi, H.Uyama, S.W.Lee and Y.Matsumoto, J. Polym. Sci., Polym. Chem. Ed,, 31, 3133 , (1993) 